\author{
DEPARTAMENTO DE FISIOLOGIA DA FACULDADE DE MEDICINA DA \\ UNIVERSIDADE DE. SAO PAULO \\ Diretor: Professor Frantilin A. de Moura Campos
}

\title{
INFLUENCIA DE ALGUMAS DIETAS SOBRE O METABOLISMO BASAL (*)
}

\section{DEMOSTHENES ORSINI}

Assistente de Fisiologia

No presente' trabalho apresentamos os valores do metabolismo basal encontrados para ratos brancos, recebendo certas dietas geralmente usadas em pesquisas sobre Nutrição. Assim procuramos conhecer o papel dessas dietas na produção calórica básica desses animais. Tambem citamos resultados obtidos com pacientes humanos, em condições diversas de nutrição.

Este trabalho faz parte de uma serie de observações, já publicadas ou em vias de publicação, que realizamos sobre o Metabolismo Basal em condições várias.

A alimentação como fator capaz de influir sobre o valor do metabolismo de base é reconhecida por inumeros autores. A super-alimentação póde condicionar aumento da despeza de base, em animais (1-2 e em pacientes humanos (3-4). A redução do metabolismo de base como um dos indices de desnutrição de um povo é um problema de capital importância sob todos os seus aspectos. Os baixos valores do metabolismo basal encontrados em nosso meio por inumeros autores nacionais (5) devem ser atribuidos, pelo menos em parte, ao nosso regime alimentar incompleto e desharmonico, reconhecido por vários pesquisadores (6-7).

A redução do metabolismo de base observada no sul dos Estados Unidos foi atribuida ao menor consumo de proteinas dessas populações (8-9).

Um perene estado de desnutrição dos povos orientais com todas as suas consequencias funestas, é reconhecido por inumeros autores (10-11-12-13-14-15-16). E' justamente no Oriente que se encontra com mais constancia a redução do metabolismo basal, explicavel pela deficiência alimentar (17-18-19-20-21).

(*) Agradecemos muito sinceramente aos Professores Drs. E. B. Hart, C. A. Elvehjem, da Universidade de Wiscousin, e Dr. J. R. Murlin, da Universidade de Rochester, em cujos laboratorios réalizamos a.maior parte deste trabalho. 
Em trabalho anterior (22) usando um pequeno numero de ratos brancos e em condições experimentais diversas, encontramos a redução do metabolisino de base dos animais que receberam uma dieta, qualitativa ou quantitativamente, deficiente em proteinas. Para jovens brasileiros, de 8 a 18 anos de idade, verificamos (23) certa baixa do metabolismo basal que atribuimos, em parte, à incompleta alimentação que recebiam.

\section{MATERIAL}

Para as nossas observações usamos 47 ratos brancos, machos, divididos em 4 grupos de acordo com a dieta. Tanto quanto possivel, pois as observações foram realizadas em dois laboratorios e em diferentes epocas, procuramos conservar identicas condições experimentais quanto à idade, cuidados preliminares, etc. Não levamos em consideração a possivel influencia estacional porque os animais foram criados em ambiente de ar condicionado e as provas realizadas a $28^{\circ} \mathrm{C}$, temperatura ótima para a determinação do metabolismo basal de ratos (24). Devido a esse criterio de uniformidade cuidamos que os resultados dos diversos grupos são suscetiveis de comparação entre si, embora, a rigor, a comparação deva ser feita entre os diversos subgrupos dum mesmo grupo.

Da normalidade dos animais, avaliamos pelo aspecto geral, curva ponderal, apetite e ausencia de sinais indicativos de anormalidade.

O primeiro grupo foi dividido em 6 sub-grupos de acordo com as rações iso-caloricas $\mathrm{R} 119-120-121-122-123-124$ que recebiam (Quadro I). Assim procuramos estudar o papel das gorduras e hidratos de carbono em diferentes percentagens. Em maior proporção usamos ou gordura de porco ou gordura vegetal ("Crisco" - gordura vegetal largamente usada nos Estados Unidos).

No segundo grupo procuramos estudar, usando as rações $R$ 125126, a influencia do amido e da sucrose em taxas identicas (Quadro I). O amido, alem de se distinguir sob o ponto de vista alimentar por diversos aspectos, tem ação bem diversa sobre a flora intestinàl, fator importante na sintese de algumas vitaminas.

No terceiro grupo foram usadas as rações R 127-128-192-130 (Quadro I) que diferem essencialmente entre si pelas taxas de proteinas, no sentido de que as rações modificadas encerram uma taxa proteica excessiva pois, como mostraram Mendel e Osborne (25), $20 \%$ de proteina é a quantidade necessaria para o rato.

No quarto grupo (Quadro II), onde usamos as rações $\mathrm{R}$ 131132 , procuramos estudar a influencia duma ração lactea quando comparada com uma dieta bem equilibrada ("Purina Laboratory Chows" - ração comercial largamente utilisada nos Estados Unidos para alimentação de animais de laboratorio) como provam numerosas observações (26). Ao leite cuja composição damos no Quadro III, eram adicionados sais minerais para compensar a sua pobreza nesses elementos. A cada 15 c. c. 'de leite eram adicionados 15 c. c. de agua, 


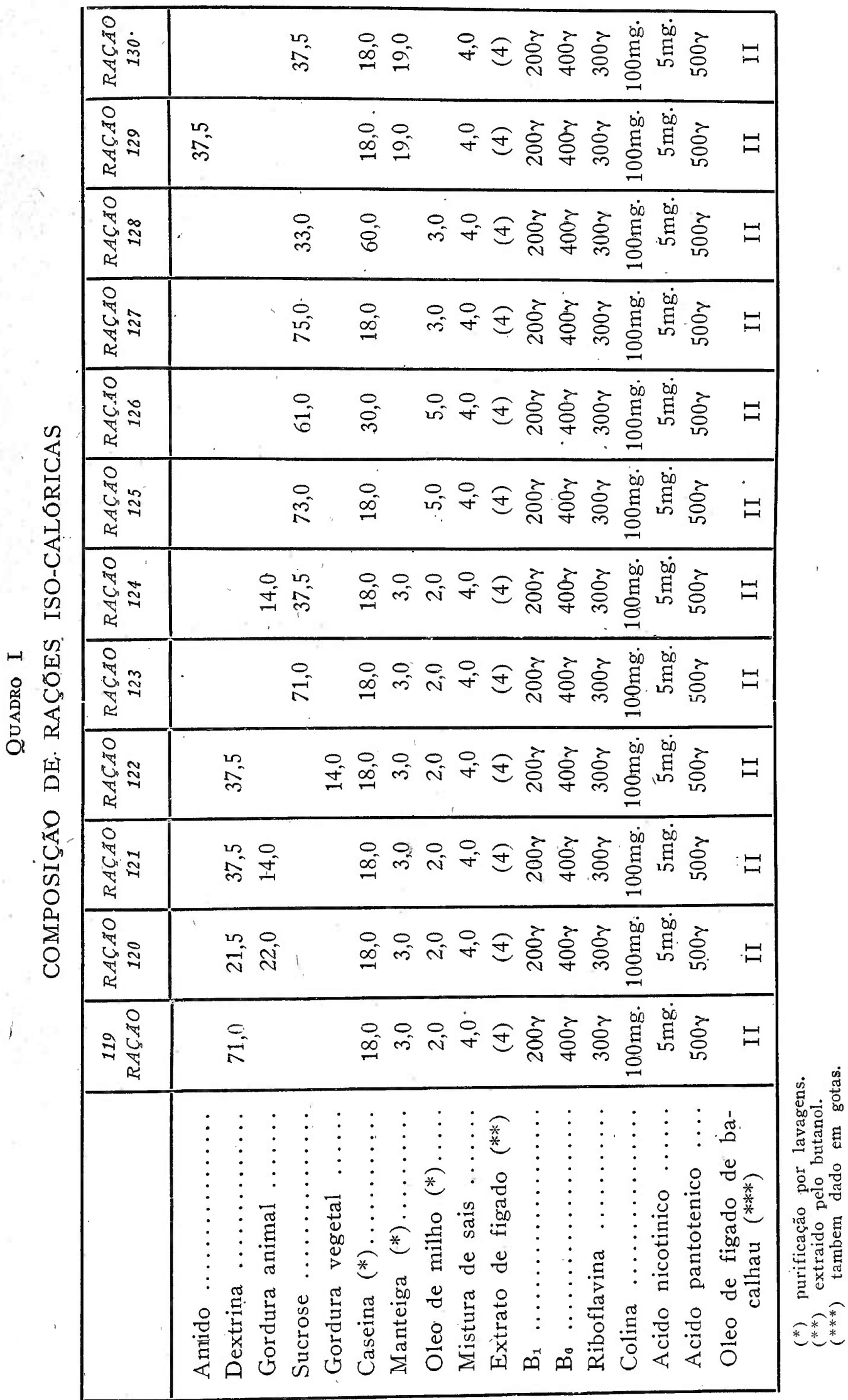


0,5 mg. de Fe, 0,04 mg. de Cu, 0,05 mg. de Mg e 245 U. S. P. de vitamina $\mathrm{D}$.

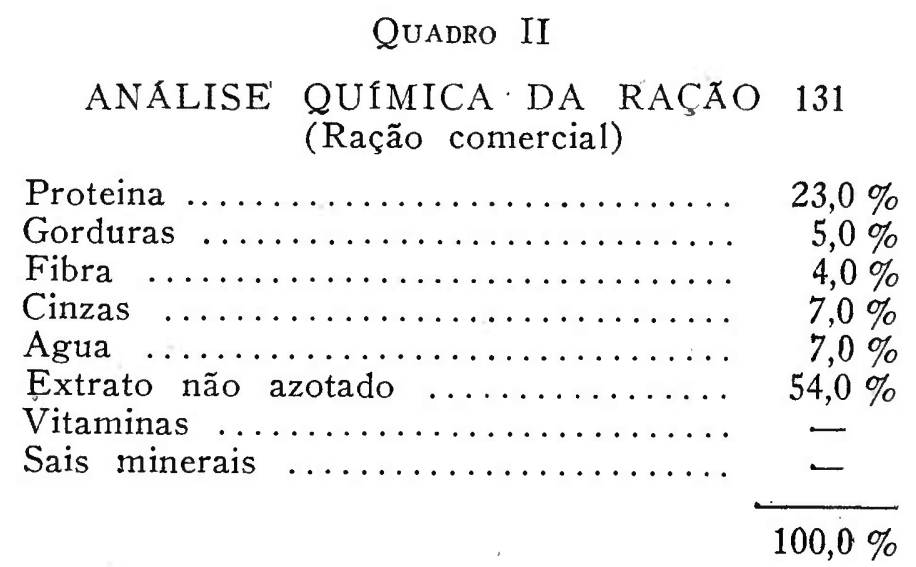

QUADro III

COMPOSIÇÃO MÉDIA DA RAÇÃO 132

(Leite evaporado)

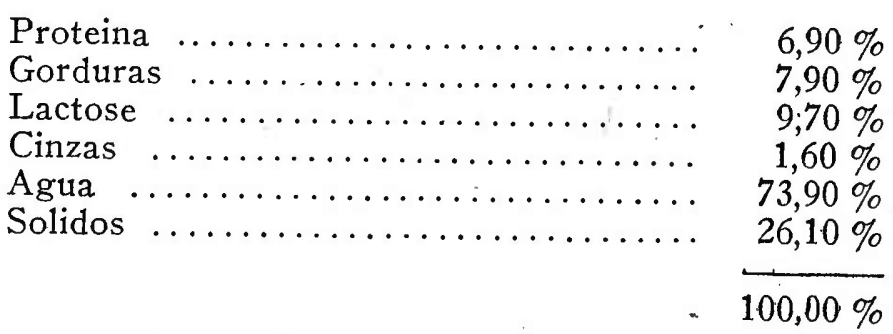

Os nossos pacientes humanos compreendem dois grupos de jovens do sexo masculino, de 14 a 18 anos de idade. O primeiro grupo compreende 110 pacientes recolhidos, recentemente, a um Abrigo de Menores da cidade de S. Paulo. $\mathrm{m}$ inquerito levado a efeito entre 211 rapazes antes de serem abrigados, revelou o seguinte:

\section{Condições \\ alimentares}

$\begin{array}{lr}\text { bôas } \ldots \ldots \ldots \ldots \ldots \ldots \ldots & 32,22 \% \\ \text { más } \ldots \ldots \ldots \ldots \ldots \ldots & 38,87 \% \\ \text { regulares } \ldots \ldots \ldots \ldots \ldots & 19,90 \% \\ \text { ignoradas } \ldots \ldots \ldots \ldots \ldots & 9,00 \%\end{array}$

O segundo grupo compreende 139 pacientes recolhidos, durante um tempo relativamente longo, ao mesmo Abrigo de Menores, onde recebem alimentação abundante e variada. Em trabalho anterior (27) assinalamos, ao lado do desigual estado de nutrição, a maior atividade muscular dos componentes deste segundo grupo.

\section{TECNICA}

Usamos dois metodos de calorimetria, um indireto e outro direto.

As provas de animais dos tres primeiros grupos foram feitas com o aparelho construido por Schwabe e Griffith (28) e cuja técnica 
seguimos. Trata-se dum aparelho de circuito fechado que utilisa oxigenio puro, cujo consumo é dado pelo desnivel dum estilete que inscreve sobre um quimografo em movimento. O gaz carbonico eliminado é absorvido por' uma solução de agua de barita, cujo titulo é determinado antes e depois de cada prova, dando, assim, a quantidade de $\mathrm{CO}_{2}$ produzida.

$\mathrm{Na}$ determinação do metabolismo basal dos animais do quarto grúpo utilisamo-nos da camara calorimetrica construida por Murlin e Barrowns (29) e cuja técnica seguimos. A análise do ar ambiente e dos gazes que saem da camara é feita pelo aparelho de Haldane, dando-nos, assim, o consumo de $\mathrm{O}_{2}$ e a produç̧ão de $\mathrm{CO}_{2}$. $\mathrm{O}$ vapor dagua produzido é absorvido por um frasco com acido sulfurico, cuja variação de peso nos indica a quantidade de $\mathrm{H}_{2} \mathrm{O}$ eliminada.

A produção calórica direta é registrada, eletricamente, em um quimografo especial.

Assim este método associa a calorimetria indireta e direta.

Os cuidados durante as provas e os que precedem a realização das mesmas foram seguidos de acordo com as indicações dos citados autores e sob o mesmo criterio seguido por nós em trabalhos semelhantes (30).

Os animais foram pesados imediatamente depois da realização das provas, portanto em jejum. Para o calculo da area da superficie corporal usamos a formula de Diack (31) para maior uniformidade em relação aos autores américanos.

As provas foram realizadas entre as 10 e 16 horas quando, segundo Benedict (32) se observa melhor repouso dos ratos. Realizamos para cada animal sempre mais de uma prova, considerando nos nossos calculos os valores mais baixos.

Para os nossos pacientes humanos usamos o aparelho de Benedict-Roth, cuja técnica é bastante conhecida e já descrita em trabathos anteriores (33).

\section{RESULTADOS}

Os valores encontrados foram relacionados ao metro quadrado de superficie corporal e, para os ratos, ao quiligromo de peso corporal:

No Quadro IV demos esses valores e os desvios em percentagem tomando como base os valores fornecidos pelos ratos recebendo as rações $\mathrm{R} 123-125-127-131$ consideradas como basicas nos respectivos sub-grupos. Verifica-se que os nossos resultados são comparaveis aos obtidos por autores americanos para ratos normais (34). As diversas dietas usadas não influiram sobre os valores do metabolismo de base encontrados sendo que os desvios verificados núnca excederam de $\pm 10 \%$. Comparando-se as médias de 1068, 1011, 1132,1001 Cals. $/ \mathrm{m}^{2} / 24$ hs. obtidas respectivamente para o primeiro, segundo, terceiro e quarto grupo, verifica-se que elas são suficientemente aproximadas para não se considerar como significativas essas variações. 
QUADRO IV

METABOLISMO BASAL EM RATOS BRANCOS RECEBENDO DIVERSAS RAÇÕES

\begin{tabular}{|c|c|c|c|c|c|c|}
\hline \multirow{2}{*}{ N. de animais } & Peso & Q. R. & Cals./m2/24 hs. & Desvio\% & Cals./Kgm/24hs. & Rações \\
\hline 3 & 190 & 0,84 & 1.180 & $+9,0$ & 152 & $\mathrm{R} 119$ \\
3 & 130 & 0,83 & 1030 & $-4,8$ & 154 & $\mathrm{R} 120$ \\
3 & 150 & 0,82 & 1112 & $+2,8$ & 156 & $\mathrm{R} 121$ \\
3 & 148 & 0,86 & 1.008 & $-6,8$ & 141 & $\mathrm{R} 122$ \\
3 & 154 & 0,83 & 1.182 & - & 151 & $\mathrm{R} 123$ \\
3 & 145 & 0,87 & 997 & $-7,8$ & 140 & $\mathrm{R} 124$ \\
3 & 130 & 0,79 & 1.037 & - & 154 & $\mathrm{R} 125$ \\
3 & 167 & 0,81 & 985 & $-5,0$ & 135 & $\mathrm{R} 126$ \\
2 & 122 & 0,82 & 1.187 & - & 182 & $\mathrm{R} 127$ \\
4 & 210 & 0,84 & 1.148 & $-3,3$ & 148 & $\mathrm{R} 128$ \\
2 & 230 & 0,80 & 1.122 & $-5,5$ & 148 & $\mathrm{R} 129$ \\
3 & 172 & 0,81 & 1.070 & $-9,8$ & 145 & $\mathrm{R} 130$ \\
6 & 170 & 0,79 & 1015 & - & 137 & $\mathrm{R} 131$ \\
& & & & & & (ração comer- \\
cial) \\
6 & 168 & 0,78 & 987 & $-2,7$ & 135 & R 132 \\
(ração lactea) \\
\hline
\end{tabular}

No Quadro V damos os resultados obtidos para os pacientes humanos e os desvios percentuais em relação ao padrão Boothby-Sandiford. No Gráfico I projetamos os nossos valores.

Como se vê os valores médios encontrados no segundo grupo são superiores aos do primeiro grupo e mesmo aos dos padrões americanos.

Alem de outros fatores, o melhor estado de nutrição dos pacientes do segundo grupo, que pode ser bem verificado no Quadro VI, seria uma das causas explicativas dos valores mais altos encontrados.

\section{QUADRo VI}

PESOS E ALTURAS DE PACIENTES EM CONDIÇÕES DIVERSAS DE .NUTRIÇÃO

\begin{tabular}{|c|c|c|c|c|c|}
\hline \multicolumn{3}{|c|}{ Pacientes do $1 .^{\circ}$ Grupo } & \multicolumn{3}{|c|}{ Pacientes -do $2 .^{\circ}$ Grupo } \\
\hline $\begin{array}{l}\text { Idade } \\
\text { em } \\
\text { anos }\end{array}$ & $\begin{array}{l}\text { Peso } \\
\text { médio } \\
\text { em K. }\end{array}$ & $\begin{array}{l}\text { Altura } \\
\text { média } \\
\text { em M. }\end{array}$ & $\begin{array}{l}\text { Idade } \\
\text { em } \\
\text { anos }\end{array}$ & $\begin{array}{l}\text { P'eso } \\
\text { médio } \\
\text { em K. }\end{array}$ & $\begin{array}{l}\text { Altura } \\
\text { média } \\
\text { em M. }\end{array}$ \\
\hline $\begin{array}{l}14 \\
15 \\
16 \\
17 \\
18\end{array}$ & $\begin{array}{l}42,03 \\
49,95 \\
49,87 \\
51,89 \\
56,65\end{array}$ & $\begin{array}{r}1,498 \\
1,593 \\
1,583 \\
1,603 \\
1,637\end{array}$ & $\begin{array}{l}14 \\
15 \\
16 \\
17 \\
18\end{array}$ & $\begin{array}{l}42,00 \\
45,25 \\
51,43 \\
53,17 \\
60,34\end{array}$ & $\begin{array}{l}1,533 \\
1,560 \\
1,636 \\
1,637 \\
1,674\end{array}$ \\
\hline
\end{tabular}




\section{SUMARIO E CONCLUSÕES}

O autor apresenta os resultados do metabolismo basal para ratos brancos, nascido e criados em ambiente de ar condicionado, recebenco dietas várias. Foi estudada a influencia das dietas usadas quanto à quantidade e qualidade dos hidratos de carbono ( R 119 - dextrina $75 \%$ - R 123 - sucrose $75 \%$ - R 124 - sucrose 50\%, gordura de porco $25 \%$ - R 129 - amigo $50 \%$, manteiga $25 \%$ - R 130 - sucrose $50 \%$, manteiga $25 \%$ ), gorduras ( R 120 - gordura de porco $40 \%$ - R 121 - gordura de porco $25 \%$ - R 122 - gordura vegetal "Crisco" $25 \%$ ), proteina (R 125 - R 127 - Caseina $18 \%$ - R 126 - Caseina $30 \%$ - R 128 - $68 \%$ ) e outras caracteristicas ( R 131 - Ração comercial bem equilibrada - R 132 Ração lactea). As comparações foram feitas com os animais recebendo as dietas consideradas básicas.

Nas determinações do metabolismo basal foram usados métodos de calorimetria indireta (aparelho de Schwabe e Griffith) e direta (calorimetro automatico de Barrows e Murlin).

São ainda citados os valores do metabolismo basal (pelo método de Benedict-Roth) de jovens de 14 a 18 anos de idade, sexo masculino, en condições diversas de nutrição, etc.

1) Os valores obtidos para o metabolismo basal de ratos brancos, recebendo dietas várias, podem ser considerados como valores normais.

2) As dietas usadas não 'afetaram' os valores do metabolismo basal dos ratos brancos observados, pois os desvios encontrados não vão alem de $\pm 10 \%$ em relação aos animais testemunhas:

3) Foi encontrada nitida diferença entre os valores do metabolismo de base de jovens de 14 a 18 anos de idade, em condições diversas de nutrição, etc.

\section{BIBLIOGRAFIA}

1 - Grafe e Grahaim - Zeitsch f. Physiol. Chem., 1911, 1, 73.

2 - Eckstein e Grafe - Zeitsch f. Phisiol. Chem., 1919, 107, 37.

3 - Grafe, E. - Ergebenisse der Physiologie Band II, Abteiling, Munchen, 1923.

4 - Grafe e Kock - Deutsch Arch. f. Klin. Med., 1912, 106, 564.

5 - Moura Campos, F. A. - Cultura M dica, 1940, Ano II, sup. n. 1, 432.

6 - Barros Barreto, J. e Castro, A. - Arq. de Higiene do Brasil, 1938. ano 8, n. $2,399$.

7 - Paula Sousa, G. H., Cintra, A. U. e Carvalho, P. E. - Boletim n. 58, Rev. Arq. Municipal S. Paulo, 1935, n. 3.

8 - Hafkesbring, R. e Borgstrom, P. - The Amer. Journ. of Physiol., 1926, 79, 221.

9 - Williams, G. D. e Benedict, F. G. - The Amer. Journ. of Physiol., $1928,85,634$.

10 - McCay, D. - Phyllip J. Sc. Serv., 1910, 5, 163.

11 - McCarrison, R. - Race Practicioner, 1925, 114, 90.

12 - McCarrison, R. - For East A. Trop. Med., 1927, 3, 321. 
13 - McCarrison, R. - Nutrit. Abstr. Rev., 1932, 2.

14 - Aykron, W. R. - Vitamins and other dietary essentiels, 1933, Wil. Heinemann, Londres.

15 - Powell, M. N. - Chinese Physiol. Rep. Serv., n. 1, Metabolism, $1928,129$.

16 - Wu, H. e Wu, D. J. - Chinese J. Phisiol. Rep. Serv., n. 1, Metabolism, $1928,135$.

17 - Aron, H. - Phyllip J. Sc. Serv. B. 4, 1919, $192,5$.

18 - Concepcion, I. - Rev. Phyllip. Medicina, Farm., 1919, 10, 1933.

19 -- Roxas, M. L. — Phyllip Agric., 1930, 10, 121.

20 - Santos, F. C. - J. Phyllip. Islands M. A., 1930, 10, 121.

21 - Krishmann, B. T. e Varreed, C. - Indian J. Med. Res. 1932, 29, 851.

22 - Orsini, D. - An. Fac. Med. Univ. S. Paulo, 1940.

23 - Orsini, D. - O Hospital, 1940, 17, n.。 5, 791.

24 -- Schwabe, E. L., Frederick, E. E. e Griffith, F. R. - J. Nutrition, $15,199$.

25 - Mendel, L. B. e Osrorne, T. B. - Journ. Biol. Chem., 1915, 20, 351.

26 - Bittner, J. J. - Differences Observed in an Albino Strair of Mice Following a Chance in Diet, 1936.

27 - Orsini, D. - Cultura Médica, ano II, sup. n. ${ }^{\circ} 1,454$.

28 - Citação (24).

29 - Barrows, W. M. e Murlin, J. R. - Amer. Philos. Soc. Proc., 78, 483-512.

30 - Orsini, D. - An. Fac. Med. Univ. S. Paulo, 1940, 16, t. 1, 71.

31 - Diack, S. L. - J. Nutrition, 1930, 3, p. 289-296.

32 - Benedict, F. G. - Carnegie Institute of Washington, 1938, 68.

33 - Orsini, D. e Puccr, W. - An. Fac. Med. Univ. S. Paulo, 1939, 15, 113.

34. - Black, A. e Murlin, J. R. - J. Nutrition, 17, n. ${ }^{\circ}$ 4, apr. 10.

\section{EMPRESA CONSTRUTORA UNIVERSAL LTDA.}

Autorizada e Fiscalizada pelo Governo Federal - Carta Patente N.` 92

SÉDE CENTRAL: Rua Libero Badaró Ns. 103 e 107

Telegrammas: "Constructora" — Telephone: 2-4550 (Rêde particular de ligaçôes internas)

CAIXA POSTAL, 2999 - SÃO PAUlo 